International Journal of Wireless \& Mobile Networks (IJWMN) Vol. 3, No. 1, February 2011

\title{
Congestion Avoidance in IP Based CDMA Radio Access Network
}

\author{
Syed Shakeel Hashmi \\ Electronics and Communication Engineering,FST ICFAI University Dehradun,India \\ hashmi_deg@yahoo.com
}

\begin{abstract}
CDMA is an important air interface technologies for cellular wireless networks. As CDMAbased cellular networks mature, the current point-to-point links will evolve to an IP-based Radio Access Network (RAN). mechanisms must be designed to control the IP Radio Access Network congestion.

This Paper implements a congestion control mechanism using Router control and channel control method for IP-RAN on CDMA cellular network. The Router control mechanism uses the features of CDMA networks using active Queue Management technique to reduce delay and to minimize the correlated losses. The Random Early Detection Active Queue Management scheme (REDAQM) is to be realized for the router control for data transmission over the radio network using routers as the channel.

The channel control mechanism control the congestion by bifurcating the access channel into multiple layer namely RACH, BCCH and DCH for data accessing. The proposed paper work is realized using Matlab platform.
\end{abstract}

\section{Key Words}

TCP,RAN, congestion, channel control,MAC.

\section{Introduction}

The present wireless communication system is moving towards the IP enabled network, where the cellular services are integrated with IP network for the transmission of data. Such networks are generally termed as IP-RAN network. In this network the Transmission Control Protocol (TCP) is the most widely used method to achieve elastic sharing between end-to-end IP flows. At present the core network basically relies on end-system TCP to provide congestion control and sharing but this will not be acceptable in coming future because, to avoid time-out, each TCP connection requires few packets to be stored in the network, and most of that storage occurs in the router buffer which leads to congestion.

The consumers of the future will put new requirements and demands on services. However, the fact is that today we already use different kinds of multimedia services, i.e. services that to some extent combine pictures, motion and audio. These include TV, video and the Internet. Many of these applications have become fundamental elements of our lives because they fulfill basic needs, for example, communication with friends, escape from reality and last but not least simply enjoying ourselves.

As technology develops, we can satisfy these needs by using new tools, new applications and new personal devices. When utilizing these new personal tools and services to enrich our lives, while being mobile, we are using Mobile Multimedia applications. As new handsets, new technologies and new business models are introduced on the marketplace, new attractive multimedia services can and will be launched, fulfilling the demands. Because the number of multimedia services and even more so, the context in which the services are used is numerous, the following model is introduced in order to simplify and clarify how different services will evolve, enrich our lives and fulfill our desires.

DOI : 10.5121/ijwmn.2011.3107 
International Journal of Wireless \& Mobile Networks (IJWMN) Vol. 3, No. 1, February 2011

Without sufficient storage in router, the time-out will give a poor performance to the end user and prevent sharing in network. Providing larger storage for large number of connections will cause too much latency. So, if latency is to be limited then the number of connections must be severely reduced.

With the increase in the data access using these protocol, demands for larger bandwidth in coming future. Increasing bandwidth may not be a suitable solution as it is economically nonadvisable. The decrease in the resources may lead to congestion in the network resulting to complete collapsing of the network. A mechanism is hence required to overcome these problems so as to support larger data in the constraint resource to provide fair routing with least congestion.

\section{Congestion}

An important issue in a packet-switched network is congestion. Congestion in a network may occur if the load on the network i.e. the number of packets sent to the network is greater than the capacity of the network. Congestion may occur due to several reasons such as overloading the network, burst transmission, variable bit rate transmission etc. congestion reduces the performance of a network and to be controlled.

Congestion control refers to the mechanism or technique to keep the network load below the capacity limit. Congestion happens in any system due to the involvement of waiting, abnormality in the flow etc. In network congestion occurs because routers and switches have queues or buffers that hold the packets before and after processing.

For example, a router has an input queue and an output queue for each interface. When a packet arrives at the incoming interface, it undergoes three steps before departing,

1.The packet is put at the end of the input queue while waiting to be checked.

2. The processing module of the router removes the packet from the input queue once it reaches front of the queue and uses its routing table and the destination address to find the route.

3. The packet is put in the appropriate output queue and waits its turn to be sent.

The two issues which result in congestion are;

1) If the rate of packet arrival is higher than the packet processing rate, the input queues become longer and longer.

2) If the packet departure rate is less than the packet processing rate, the output queues become longer and longer.

The problem of congestion is a considerable factor in the up coming IP-RAN network, where the data between two cellular terminals are communicated using wireless and router interface. The IP-RAN is proposed to be integrated with CDMA communication system so as to enable IP access in Wireless network.

\section{Congestion Control Policies}

In this implementation two congestion control mechanisms to maximize network capacity while maintaining good voice quality: admission control, and router control mechanism are evaluated. Call admission control in current CDMA cellular voice networks is restricted to controlling the usage of air interface resources.

The principle underlying both schemes is regulation of the IP RAN load by adjusting the admission control criterion at the air-interface. the impact of router control in the form of active queue management is also evaluated. IP routers using a drop tail mechanism during congestion 
International Journal of Wireless \& Mobile Networks (IJWMN) Vol. 3, No. 1, February 2011

could produce high delays and bursty losses resulting in poor voice quality. Use of active queue management at the routers reduces delays and loss correlation, thereby improving voice quality during congestion. Using simulations of a large mobile network,

\subsection{Router Control Mechanism}

\subsubsection{Active Queue Management}

A traditional Drop Tail queue management mechanism drops the packets that arrive when the buffer is full. However, this method has two drawbacks.

Firstly, this mechanism allows a few connections with prior request to dominant the queue space allowing the other flows to starve making the network flow slower. Second, Drop Tail allows queues to be full for a long period of time. During that period, incoming packets are dropped in bursts. This causes a severe reduction in throughput of the TCP flows. One solution to overcome is to employ active queue management (AQM) algorithms. The purpose of AQM is to react to incipient congestion before the buffer overflows. AQM allows responsive flows, such as TCP flows, to react timely and reduce their sending rates in order to prevent congestion and severe packet losses.

Active Queue Management (AQM) interacts with TCP congestion control mechanisms, and plays an important role in meeting today's increasing demand for quality of service (QoS). Random Early Detection (RED), is an enhanced Algorithm employing Active Queue Management (AQM) scheme for it's realization. It is a gateway-based congestion control mechanism. An accurate model of TCP with RED can aid in the understanding and prediction of the dynamical behavior of the network. In addition, the model may help in analyzing the system's stability margins, and providing the design guidelines for selecting network parameters. These design guidelines are important for network designers whose aim is to improve network robustness. Therefore, modeling TCP with RED is an important step towards improving the network efficiency and the service provided to Internet users.

\subsubsection{Random Early Detection Router}

Random early Detection is one of the active queue management control mechanism deployed at gateways[2]. The RED gateway detects incipient congestion by computing the average queue size (Jacobson, 1998). The gateway could notify connections of congestions either by dropping packets arriving at the gateway or by setting a bit in packet headers. When the average queue size exceeds a preset threshold, the gateway drops or marks each arriving packet with a certain probability, where the exact probability is a function of the average queue size. RED gateways keep the average queue size low while allowing occasional burst of packets in the queue. Figure 1 show a network that uses RED gateway with a number of source and destination host. The RED congestion control mechanism monitors the average queue size for each output queue, and using randomization, chooses connections to notify of the congestion Transient congestion is accommodated by a temporary increase in the queue. Longer-lived congestion is reflected by an increase in the computed average queue size and result In randomized feedback to some of the connections to decrease their windows. The probability that a connection is notified of congestion is proportional to that connection's share of the throughput through the gateway. 


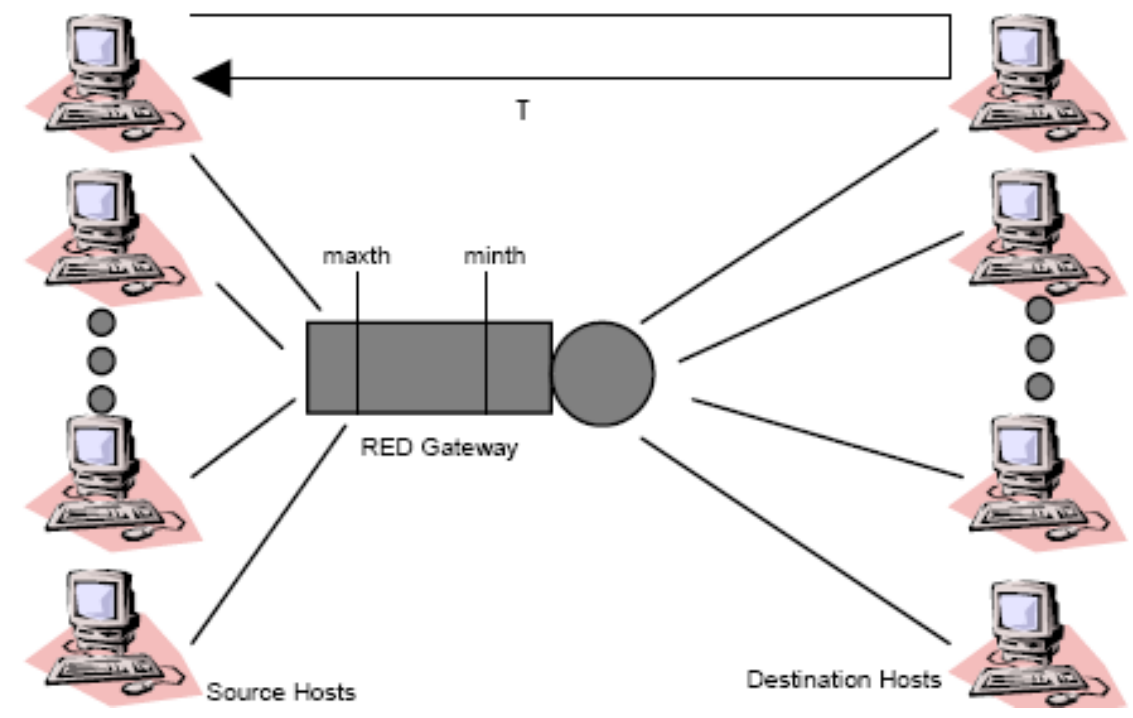

Fig:1 A network with RED gateway

\subsubsection{Red Algorithm}

RED mechanism contains two key algorithms. One is used to calculate the exponentially weighted moving average of the queue size, so as to determine the burstiness that is allowed in the gateway queue and to detect possible congestion. The second algorithm is for computing the drop or marking probability, which determines how frequently the gateway drops or marks arrival packets. This algorithm can avoid global synchronization by dropping or marking packets at fairly evenly spaced intervals. Furthermore, sufficiently dropping or marking packets, this algorithm can maintain a reasonable bound of the average delay, if the average queue length is under control.

The Random Early Detection (RED) algorithm is given as[6],

Let ' $w q$ ' be the weight factor and $q k+1$ be the new instantaneous queue size.

' $q$ ' is the average queue size and ' $q$ ' is the instantaneous queue size. Then at every packet arrival, the RED gateway updates the average queue size as

$$
\bar{q}_{k+1}=\left(1-w_{q}\right) \cdot \bar{q}_{k}+w_{q} \cdot q_{k+1} .
$$

During the period when the RED gateway queue is empty, the system will estimate the number of packets ' $m$ ' that might have been transmitted by the router. So, the average queue size is updated as

$$
\bar{q}_{k+1}=\left(1-w_{\varphi}\right)^{m} \cdot \bar{q}_{k}
$$

where

$m=i d l e \_t i m e /$ transmission_time

Where idle_time is the period that the queue is empty and transmission_time is the typical time that a packet takes to be transmitted. 
International Journal of Wireless \& Mobile Networks (IJWMN) Vol. 3, No. 1, February 2011

The average queue size is compared to two parameters: the minimum queue threshold qmin, and the maximum queue threshold qmax. If the average queue size is smaller than qmin, the packet is admitted to the queue. If it exceeds qmax, the packet is marked or dropped.

If the average queue size is between qmin and qmax, the packet is dropped with a drop probability $p b$ that is a function of the average queue size.

$$
p_{k_{k+1}}= \begin{cases}0 & \text { if } \quad \bar{q}_{k+1} \leq q_{\min } \\ 1 & \text { if } \quad \bar{q}_{k+1} \geq q_{\max } \\ \frac{\bar{q}_{k+1}-q_{\min }}{q_{\max }-q_{\min }} \cdot p_{\max } & \text { otherwise }\end{cases}
$$

where pmax is the maximum packet drop probability.

The final drop probability ' $p a$ ' is given by

$$
p_{a}=\frac{p_{b}}{1-\text { count } \cdot p_{b}}
$$

Count is the cumulative number of the packets that are not marked or dropped since the last marked or dropped packet. It is increased by one if the incoming packet is not marked or dropped. Therefore, as count increases, the drop probability increases. However, if the incoming packet is marked or dropped, count is reset to 0 .

\subsubsection{Red Method}

For each packet arrival

calculate the average queue size avg

if minth $<=$ avg $<$ maxth

calculate the probability $p a$

with probability $p a$ :

mark the arriving packet

else if maxth $<=$ avg

mark the arriving packet.

\subsubsection{Red Drop Probability $\left(\mathbf{P}_{\mathrm{a}}\right)$}

$p b=\operatorname{maxp} \times($ avg - minth $) /($ maxth - minth $)[3.5]$

where

$$
p a=p b /(1-\text { count } \mathrm{x} p b) \text { [3.6] }
$$

$p b=p b \times$ PacketSize/MaxPacketSize [3.5a] 


\subsubsection{Avg -Average Queue Length}

$a v g=(1-w q) \times a v g+w q \times q$

Where $\boldsymbol{q}$ is the newly measured queue length.

This exponential weighted moving average is designed such that short-term increases in queue size from bursty traffic or transient congestion do not significantly increase average queue size.

\section{2 channel control method}

A medium access control (MAC) protocol is developed for wireless multimedia networks based on frequency division duplex (FDD) wideband code division multiple access (CDMA).

In order to admit real-time connections, a Connection Admission Control (CAC) scheme is needed. One of the challenging issues of a CAC algorithm is that the bandwidth requirement of a connection cannot be determined at connection admission time due to the traffic burst. Thus, effective bandwidth-based CAC schemes are generally used to solve this problem.

\subsubsection{Channel Admission Control (CAC)}

The congestion occurring in CDMA can be controlled by proper controlling of channel accessing using the channel control methods[12],[13]. For the controlling of channel accessing in cdma 2000 a MAC protocol is proposed. In the MAC protocol following are the transport channels used:

1) Random access channel (RACH) is used by mobile terminals to send control packets,

2) Broadcast control channel $(\mathrm{BCCH})$ conveys system information from the base station to mobile terminals, and

3) Dedicated channel(DCH) is a point-to-point channel used to transmit data from mobile terminals to the base station or vice versa. The different transport channels are multiplexed in the code division.

Many transport channels are defined for wide-band FDD-CDMA networks in 3GPP specifications. The packet transmission in dedicated channels (DCHs) is focused in this implementation. Since signaling channels are required to assist DCHs, I also consider two other transport channels, i.e., the random access channel (RACH) to send requests from a mobile terminal to the base station and the broadcast channel $(\mathrm{BCH})$ to send feedback of resource allocation from the base station to mobile terminals. Mappings of $\mathrm{DCH}, \mathrm{BCH}$, or RACH onto a physical channel.

\section{Design Approach}

The implementation is made on the communication system following cdma architecture[5]. The transmitter and the receiver section is linked via wireless access using wireless medium and the router unit. Figure 2 shows the proposed link model for CDMA IP RAN access network. 
International Journal of Wireless \& Mobile Networks (IJWMN) Vol. 3, No. 1, February 2011

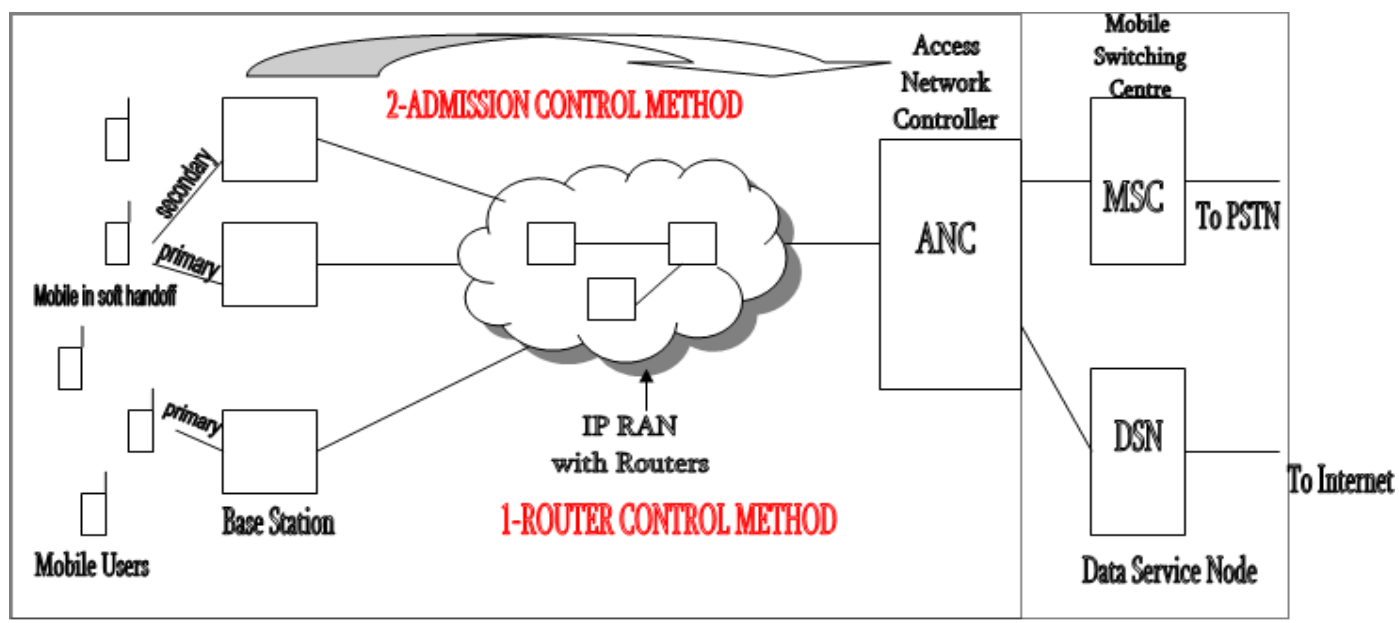

Wireless Access Network

Fig:2 CDMA Wireless Access Network with IP RAN

\subsection{MAC Controller}

The MAC controller module controls the channel access by dividing the channel into three subchannelas;

- Random access channel (RACH). This channel is used by mobile terminals to send control packets, e.g., connection requests.

- Broadcast control channel $(B C C H)$. This channel is a point-to-multipoint channel, which is used to convey system information from the base station to mobile terminals. For example, the feedback about resource allocation is transmitted in this channel.

- Dedicated channel $(D C H)$. The DCH is point-to-point channel, which is used to transmit data from mobile terminals to the base station or vice versa. DCHs, RACH and BCCH are multiplexed in the code division. In DCHs of FDD mode wideband CDMA, both MC transmission and VSF transmission are used. A DCH can have variable transmission rate depending on the spreading factor, and the basic transmission rate of the $\mathrm{DCH}$ corresponds to the maximum spreading factor used in this channel. Thus, a variable-length MAC packet is accommodated in a DCH.

\subsubsection{MAC Transmitting Operation}

When a mobile terminal wants to communicate, it needs to send a connection request through $\mathrm{RACH}$ to the receiver terminal. Once this request is received at the receiver base station, based on the availability of the receiver an acknowledgment is generated through $\mathrm{BCCH}$ sending a high signal to the transmitter unit. On reception of acknowledgment the transmitter generates a data transmission request, and on confirmation the data is transmitted through DCH channel. 
International Journal of Wireless \& Mobile Networks (IJWMN) Vol. 3, No. 1, February 2011

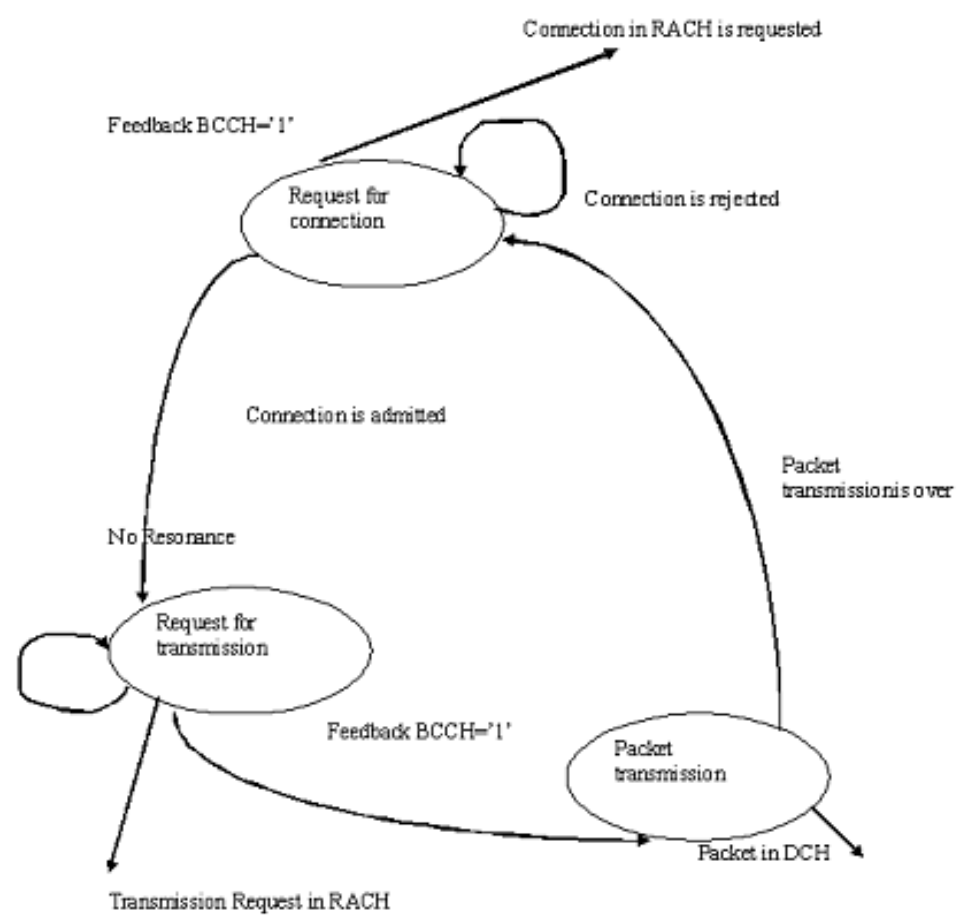

Fig:3 The operation of the mac controller is illustrated as state diagram

\subsubsection{MAC Receiving Operation}

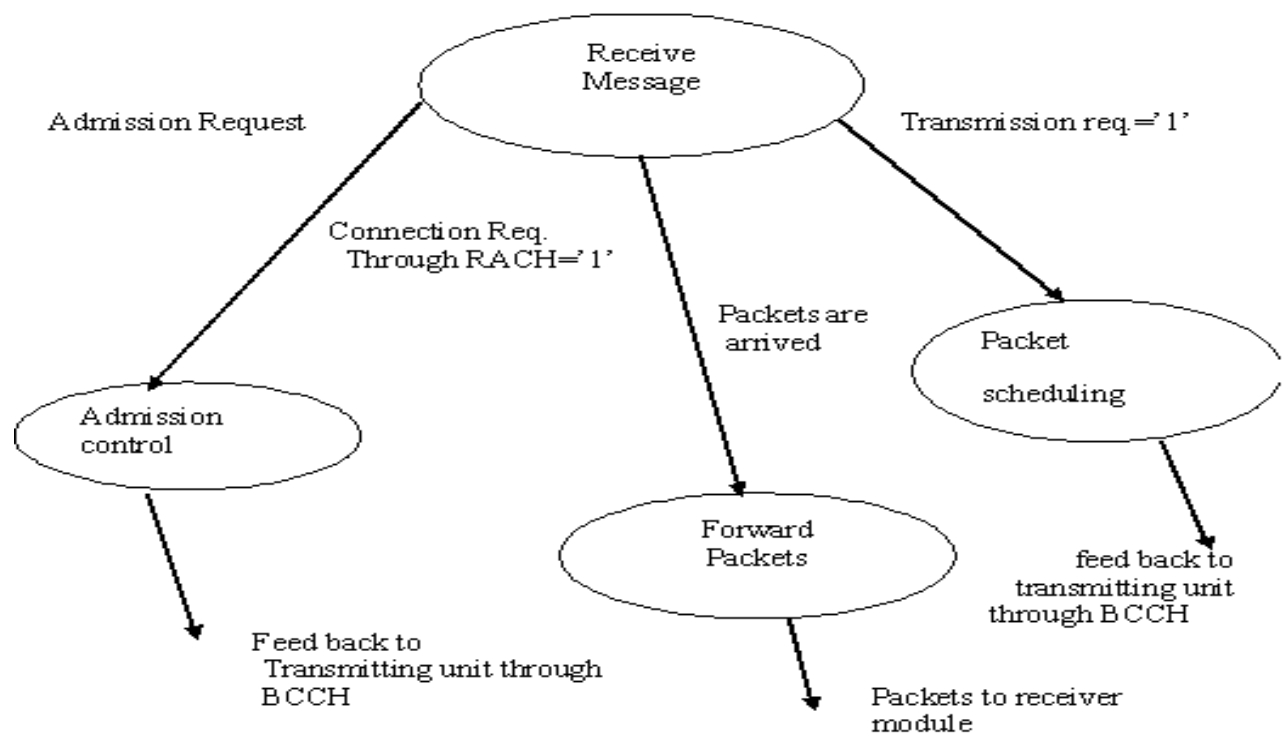

Fig:4 MAC Receiving Operations

The receiver controls the accessing using an state diagram as shown in figure 4 . The receiver respond to the request signal generated from the transmitting section and accept the data from transmitter forwarding to the receiver unit using he CAC control mechanism. 


\subsection{Router Module}

This section gives the architecture designed to implement the buffer management algorithm of Random Early Detection (RED). The architecture consists of four main components: Main Controller, Packet Drop Probability Unit (PDPU), Random Packet Drop Unit (RPDU) and the Compute Random Value Unit (CRVU). Figure shows a system architecture overview

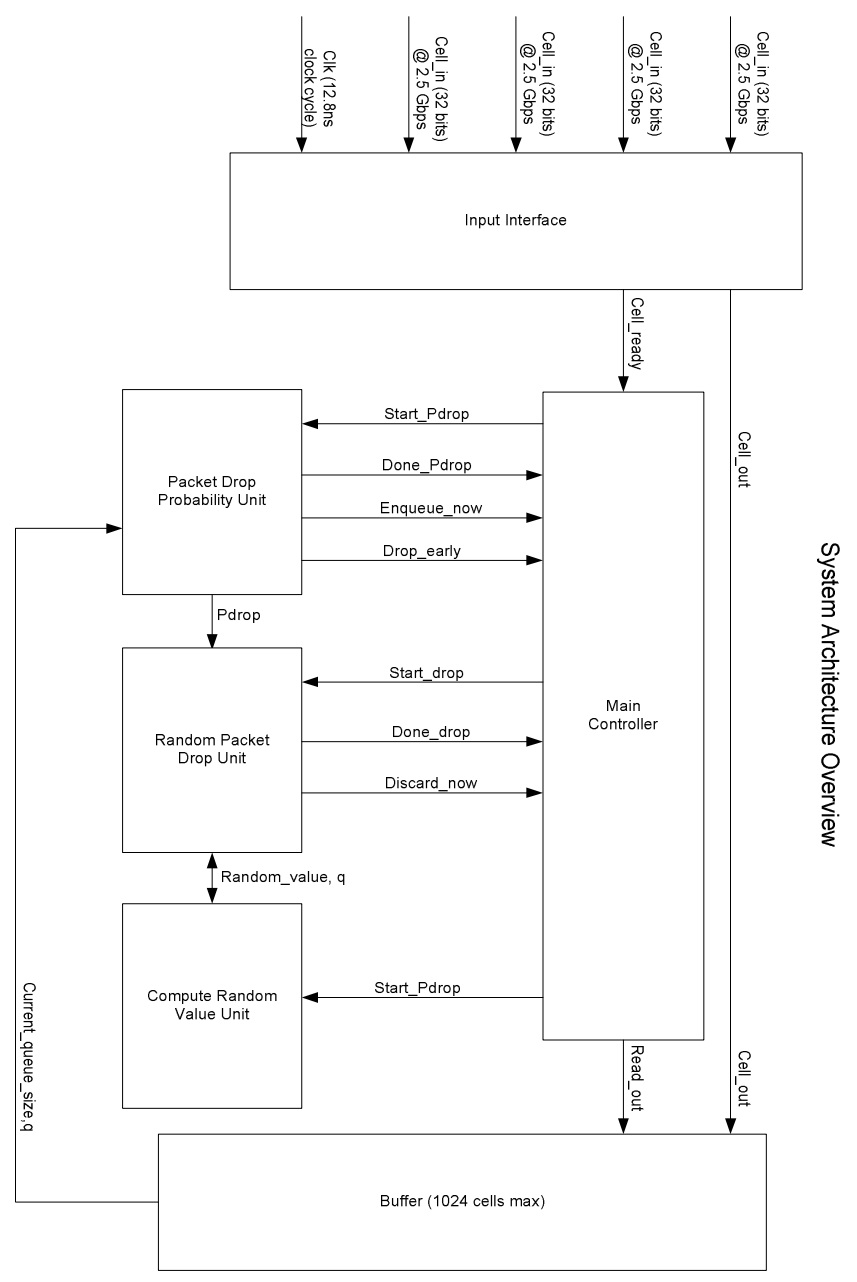

Fig:5 RED System Architecture Overview

\subsubsection{Input Interface}

RED is essentially a packet-discard algorithm. There are four concurrent connections to the input interface as shown in figure 5. One cell arrives at an input connection every four clock cycles i.e. a total of four cells arrive at the interface. Sixteen clock cycles constitute a time slot. Four cells arrive at one input connection every time slot. Thus 16 cells arrive at the input interface every time slot.

\subsubsection{Main Controller}

The Main Controller controls the entire system. It issues signals that control the Packet Drop Probability Unit, Compute Random Value Unit and Random Packet Drop Unit. The Main Controller is activated only when a cell arrives at the input interface and a cell_ready signal is issued by it. 
International Journal of Wireless \& Mobile Networks (IJWMN) Vol. 3, No. 1, February 2011

In addition, the Main Controller is responsible for interacting with the Buffer. It updates the current queue size every time slot. Two cells leave the buffer every time slot and the Main Controller reduces the current queue size by two every sixteen clock cycles. Moreover when a packet has been determined to be enqueued the current queue size is incremented.

\subsubsection{Packet Drop Probability Unit}

This module calculates the probability from the obtained q- size and decides whether to accept or drop the packets received. PDPU consists of three look up tables. The first look up table contains computed values of the average queue size, avg $\leftarrow(1-w)^{\mathrm{m}}$ avg for corresponding packet transmission time, $\mathrm{m}$. The second contains the initial packet drop probability, $\mathrm{p}_{\mathrm{b}} \leftarrow \max _{\mathrm{p}}$ $\left(\operatorname{avg}-\min _{\text {th }}\right) /\left(\max _{\text {th }}-\min _{\text {th }}\right)$ for corresponding values of avg. And the third look up table includes final packet drop probability,

$\mathrm{p}_{\mathrm{a}} \leftarrow \mathrm{p}_{\mathrm{b}} /\left(1-\right.$ count $\left.\mathrm{p}_{\mathrm{b}}\right)$, for corresponding values of $\mathrm{p}_{\mathrm{b}}$.

\subsubsection{Configure Random Value Unit}

The Configure Random Value Unit uses a random generator function to compute a random number. An 8 bit initial seed is used and set equal to "00000010". Tests were conducted with different seeds and a seed of "00000010" produced the most unbiased random number i.e. random numbers were never repeated, a unique one was output each time..

A feedback signal is computed by running an XOR process on each of 8 bits of the initial seed in the following manner:

\subsubsection{Random Packet Drop Unit}

One point to note here is the fact that only packets that were determined by the PDPU to have an average queue size between the minimum and maximum thresholds are marked. Other packets do not interact with this unit they are chiefly concerned with the PDPU.

\section{Analysis Of Complete System}

For the analysis of the complete system we need the below flowchart

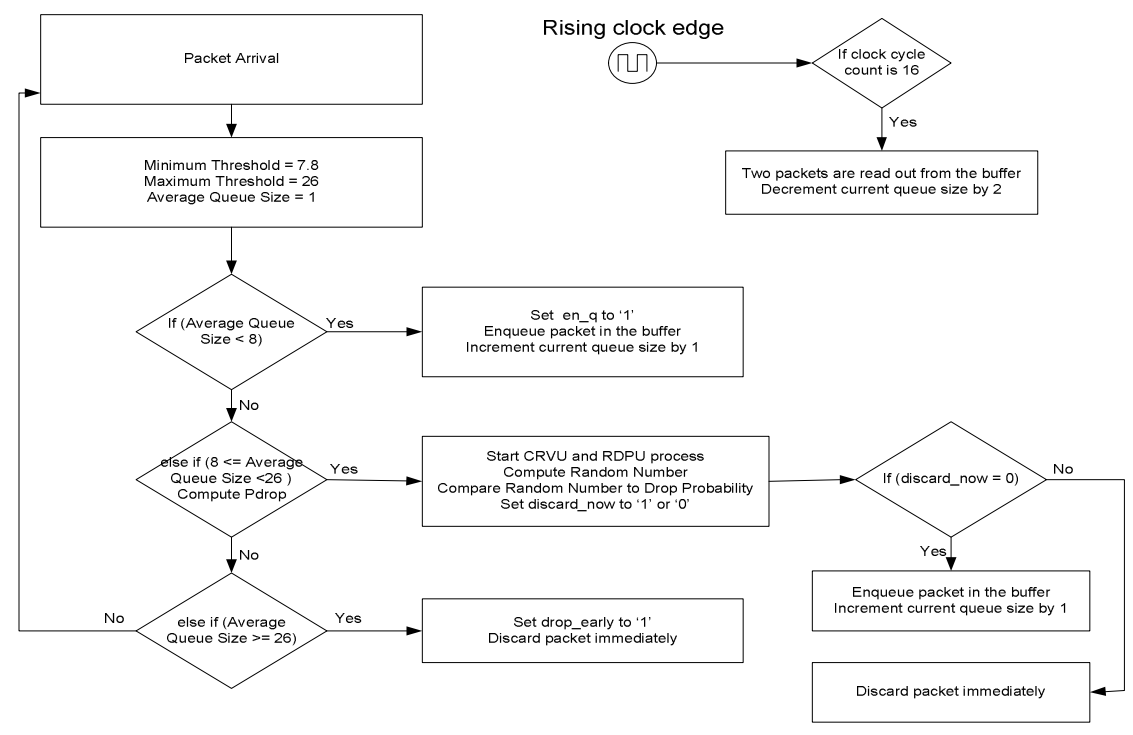

Figure 6 Summary of all processes in the RED implementation 
International Journal of Wireless \& Mobile Networks (IJWMN) Vol. 3, No. 1, February 2011

Figure6 shows that whenever count reaches 16 i.e. a time slot is over, two packets from the buffer are read out and thus the current queue size of the buffer is reduced by two. In the first case when the clock count reaches 16 the current queue size decreases by 2 .

\section{Results And Analysis}

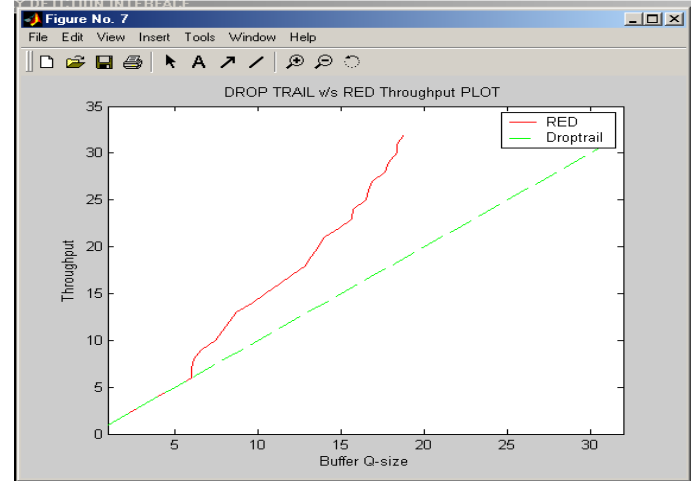

Fig: 7 Throughput v/s Q-size for Droptail and RED method

Fig illustrates the throughput performance of the Router control mechanism for RED and Droptail method. It can be observe that the throughput remain same for RED \& DT until minimum threshold.After min threshold the throughput of RED router is seem to be improved because of packet dropping .

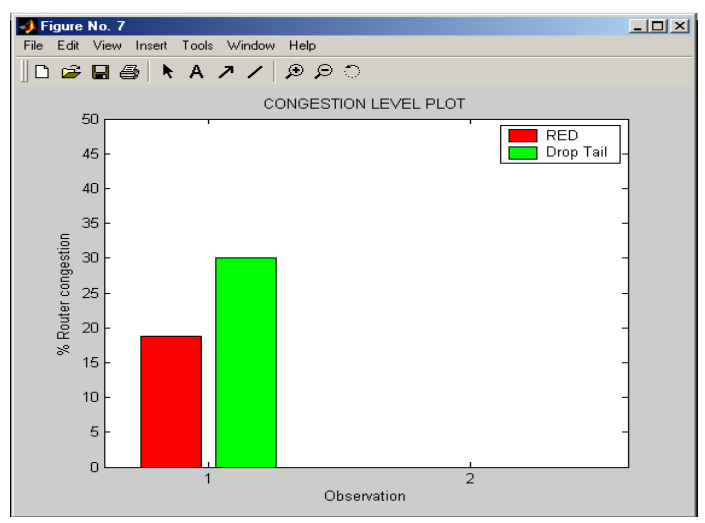

Fig:8 The congestion level plot for Droptail and RED method

Fig shows the congestion level obtained for the simulation of implemented design.It can be seen that RED gives congestion avoidance of about $40 \%$ compare to the Droptail method.

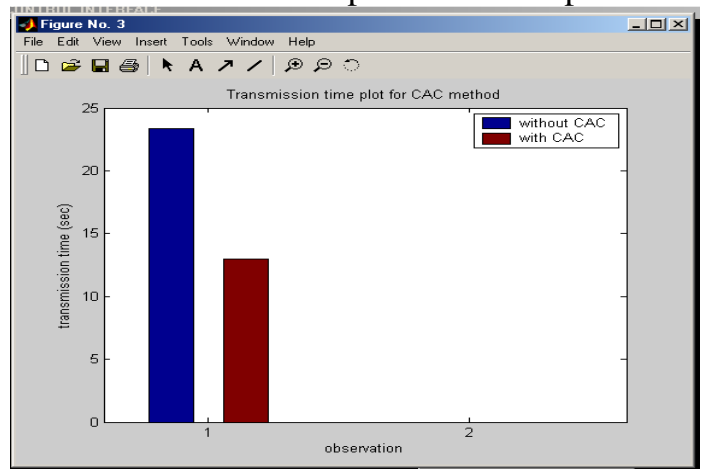

Fig: 9 Transmission time comparisons for CDMA base comm system with and without CAC 
Fig shows the transmission time comparison plot for two methods used for controlling the congestion in Air interface.From the plot it can be observed that about 50\% of transmission time could be saved using CAC method than the existing method.

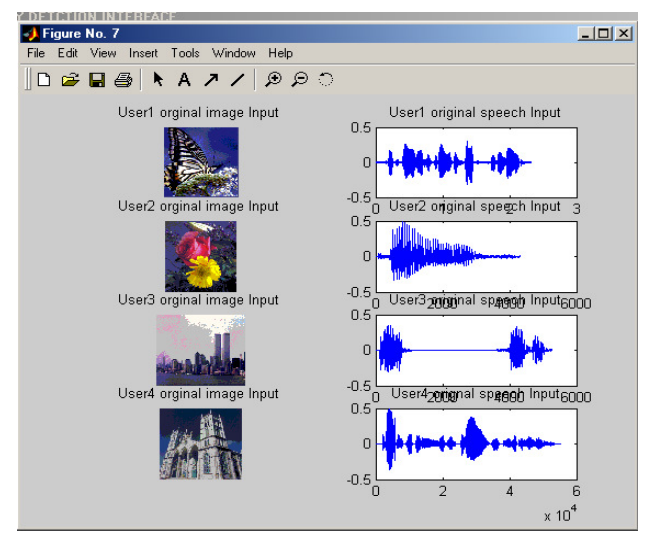

Fig:10 The image and speech samples considered for processing and evaluating the implemented system.

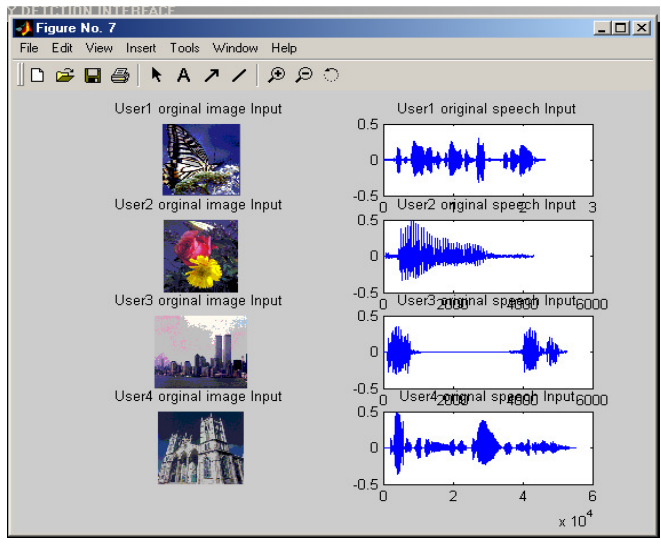

Fig11 Retrived outputs of image and speech sequence for four users

\section{CONCLUSION}

The evolution of wireless communication for multi bit rate application has come down to integration of different technologies for efficient transmission of data over wireless network.

The IP-RAN network is one such result of this technology integration. The evolved IP network though proposed to support multi bit rate application can fails in providing efficient performance for bursty network. The network fails to provide better performance due to limitations and resource such as bandwidth resulting in congestion.

This paper realizes the two most advanced congestion control policies namely the Channel Admission Control and Router Control controlling in wireless network. For the evaluation of control policies a wireless network with CDMA2000 standard is adapted.

The system realizes follows the CDMA2000 standard with CDMA transceiver architecture. The transceiver realizes are integrated with the Router and Channel control mechanism to overcome the congestion happening at the network. The CDMA architecture realized for communication is modular approached with modules such as scramble code 
International Journal of Wireless \& Mobile Networks (IJWMN) Vol. 3, No. 1, February 2011

generator using LFSR logic spreader spreading the user data over the entire bandwidth for the BW spreading of user data a OVSF spread code is realized using Walsh Hadamard coding. The multiple spreaded user data are then bundle over power spectrum modulated using BPSK modulation before transmission .A multi-user detection logic is realized at the receiver end using matched filter concept to the isolation of user data before dispreading to retrieve back the final data.

A router interface is realized as an intermediate interface between the source and destination to provide better services using an IP- enabled wireless. The congestion control policy for router control mechanism is realized using the implementation of RED algorithm. This algorithm is designed an incorporated with router architecture for the control of congestion at early stage. The controlled is achieved by floating the congestion level between minimum and maximum threshold.

An air interface control mechanism is also realized for the control of congestion occurring at the wireless channel. To avoid the congestion at channel the channel access mechanism is developed. In this mechanism the channel is bifurcated into dedicated layers using frequency division duplex, where each layer of a channel is dedicated for a particular operation named as $\mathrm{RACH}, \mathrm{DCH}$ and BCCH.For the evaluation of two algorithms, the algorithms are compared with their existing control methods.

From the implementation and observation it is seen that the performance can be improved for IP-RAN with the integration Router and Channel control mechanism from its counterparts.

The Router control mechanism can be embedded with the transceiver architecture provides 30 to $40 \%$ reduction in congestion level at the router making the transmission of the image and speech data faster. It is observed that Router design with RED algorithm provides better throughput than the Droptail method. The Channel Admission Control method realized shows a performance enhancement of about $50 \%$ faster than the earlier system.

From all the observation made, it could be concluded that CDMA system with Router control mechanism using RED algorithm and Channel control mechanism using MAC protocol can enhance the performance of the data transmission for both image and speech in wideband CDMA network.

\section{FUTURE SCOPE}

The implementation make few assumptions for the implementation of the two control mechanisms such as idle channel equal speed traffic balanced, burstiness etc. These assumptions hold well during algorithm evaluation but may deviate in its operation in real time scenario. An effort could be made to evaluate the performance considering these factors also.

The proposed implementation is evaluated on apart of image and speech sample, an effort can be made to speed up the operation of this implementation in future.

The algorithm can also be tried out with other communication system such as MC-CDMA, CSCDMA etc.

The implementation can also be evaluated considering various channel parameters for real time evaluation.

\section{REFERNCES}

1. Andreas F. Molisch,"Wideband Wireless Digital Communications”, Pearson Education, 2001, PP-18.

2. A. A. Akintola, G. A. Aderounmu,and L. A. Akanbi Obafemi Awolowo University, Ile-Ife, Nigeria, M. O. Adigun University of Zululand,Kwadlangezwa, Republic of South Africa," Modeling and 
International Journal of Wireless \& Mobile Networks (IJWMN) Vol. 3, No. 1, February 2011

Performance Analysis of Dynamic Random Early Detection (DRED) Gateway for Congestion Avoidance”, Issues in Informing Science and Information Technology,PP-623-633.

3. Behrouz A. Fourouzan,'Data Communication and Networking”, third edition, Tata McGRAWHILL, 2004.

4. Erik Dahlman, Per Beming, Jens Knutsson, Fredrik Ovesjo, Magnus Persson, and Christiaan Roobol," WCDMA-The Radio Interface for Future Mobile Multimedia Communications", IEEE TRANSACTIONS ON VEHICULAR TECHNOLOGY, VOL. 47, NO. 4, NOVEMBER 1998, PP$1105,1115$.

5. G. Heijenk, G. Karagiannis, V. Rexhepi, and L. Westberg, "Diffserve resource management in ip-based radio access networks," in Proceedings of WPMC, Aalborg, Denmark, September 2001.

6. Sneha Kumar Kasera, Ramchandran R, Sandra and Xin Wang," Congestion Control Policies for IPBased CDMA Radio Access Network,"IEEE Trans on mobile computing, vol4, pp.349-62, july/aug2005.

7. S.Floyd "Connections with multiple congested gateways in packet switched network. Part I.One way traffic”. Comput. Commun. Rev. vol.21 no.5 pp 30-47. Oct.1991

8. S.Floyd and V.Jacobson,"Random Early Detection Gateways for Congestion Avoidance", IEEE/ACM Trans.Networking, vol.1, no.4, pp.397-413, Aug1993.

9. Theodre S. Rappaport, Joseph C. Liberty," Smart Antennas for Wireless Communications IS-95 and third generation CDMA Applications", Prentice Hall PTR.

10. Vijay K. Garg,’Wireless Network Evolution”, Pearson Education, 2003.

11. Vijay K. Garg,’IS-95 CDMA and cdma2000”, Pearson Education, 2002.

12. Xudong Wang, Member, IEEE," Wide-Band TD-CDMA MAC With Minimum- Power Allocation and Rate- and BER-Scheduling for Wireless Multimedia Networks" IEEE/ACM TRANSACTIONS ON NETWORKING, VOL. 12, NO. 1, FEBRUARY 2004, PP.103,105.

13. Xudong Wang, Member, IEEE," An FDD Wideband CDMA MAC Protocol with Minimum-Power Allocation and GPS-Scheduling for Wireless Wide Area MultimediaNetworks",IEEETRANSACTIONSONMOBILECOMPUTING， VOL. 4, NO. 1, JANUARY/FEBRUARY 2005,PP-16,24

14. "www.Ericsson.com" Ericsson Radio Systems AB," Basic Concepts of WCDMA Radio Access Network", white paper.

\section{Authors}

S.Shakeel Hashmi received his B.E. Degree in Electronics \&Telecommunication Engineering from B.A.M.U.University India in 1999; $\mathrm{He}$ has obtained M.E. in Electronics \& Communication Engineering from Osmania University, Hyderabad, India.He is currently working as a faculty member in the dept. of Electronics and Communication Engineering at ICFAI University Dehradun India. His area of interest is Wireless Communication, Heterogeneous wireless networks.

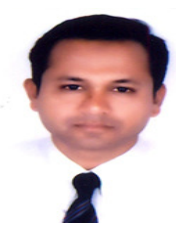

